\title{
Linked Data for science and education
}

\author{
Carsten Keßler ${ }^{\mathrm{a}}$, Mathieu d'Aquin ${ }^{\mathrm{b}}$ and Stefan Dietze ${ }^{\mathrm{c}}$ \\ a Institute for Geoinformatics, University of Münster, Germany \\ E-mail: carsten.kessler@uni-muenster.de \\ b The Open University, UK \\ E-mail:m.daquin@open.ac.uk \\ ${ }^{\mathrm{c}}$ L3S Research Center, Germany \\ E-mail:dietze@l3s.de
}

Sharing of resources, resource metadata, and data across the Web is a central principle in scientific and educational contexts. Scientific collaboration has long been striving for wider reuse and sharing of knowledge and data. Likewise, the Open Educational Resources community has promoted the widespread exploitation of public and reusable educational resources throughout the last decade. Hence, technologies to enable interoperability of shared resources and data have long been at the centre of scientific and educational information systems. However, due to the lack of a shared technology stack and joint principles, the landscape of developed and utilised standards is very fragmented and covers an increasing variety of heterogeneous technologies, such as repositories with proprietary interfaces and query mechanisms. Moreover, a broad range of largely incompatible metadata schemas and taxonomies have been developed to describe and expose educational resources, and scientific workflows and data. Due to the prevailing heterogeneity of deployed approaches and technologies, interoperability remains an open challenge.

At the same time, the Linked Data (LD) approach has emerged as the most recent evolution of the $\mathrm{Se}$ mantic Web, aiming at Web-scale data interoperability, it has produced an ever growing amount of data sets and schemas available on the Web. Given the proven capabilities of LD technologies towards realising Web scale data sharing and reuse, scientific and educational practitioners have started to adopt those principles. $\mathrm{Re}$ sults of such activities cover joint schemas used for describing scientific or educational resources, vast col- lections of structured data about, for instance, cultural or historic artifacts, bibliographic collections, domainspecific vocabularies capturing extensive amounts of scientific domain knowledge - where the life sciences are particularly well represented - as well as baseline technologies used to expose and integrate linked datasets.

This special issue contains four papers documenting the most recent developments in Linked Data for science and education. Focusing on the collection and annotation of scientific data, the paper Ontology of Units of Measure and Related Concepts by Hajo Rijgersberg, Mark van Assem and Jan Top shows how to annotate quantitative research data for sharing and reuse. The design choices made during the development of this OWL ontology are explained, and the application of the ontology is demonstrated with different use cases and the tools and web services built to handle them.

The paper Publishing Bibliographic Data on the Semantic Web using BibBase by Reynold S. Xin, Oktie Hassanzadeh, Christian Fritz, Shirin Sohrabi and Renée J. Miller addresses the next step in the research process, namely publishing the results and handling the publication metadata. BibBase is a system that automatically creates a number of services from standard BibTex files, providing the contents as rich Linked Data queryable through a SPARQL endpoint, as RSS feeds, and as HTML snippets, thus addressing a number of use cases for sharing and dissemination of publication metadata.

While the first two papers focus on the dissemination of scientific assets, the remaining two papers ad- 
dress the application of the Linked Data paradigm in education. The paper Personal Learning Environments on the Social Semantic Web by Zoran Jeremic, Jelena Jovanovic and Dragan Gasevic addresses the requirement for highly collaborative and interactive learning. It provides an overview of the state of the art in personal learning environments and the underlying principles. Based on their DEPTHS system, the authors discuss the design, implementation, analysis, and evaluation of such a learning environment based on Semantic Web technologies.

The special issue is rounded off by a paper that addresses the annotation of study programs. The Bowlogna Ontology: Fostering Open Curricula and Agile Knowledge Bases for Europe's Higher Education Landscape by Gianluca Demartini, Iliya Enchev,
Joël Gapany and Philippe Cudré-Mauroux introduces a multi-lingual OWL ontology that allows universities to publish their curricula according to the Bologna process, i.e., with a focus on students planning external semesters at other universities, and students switching programs after their undergraduate degree.

This special issue shows, through these four widely different but exemplary works, that the use of Semantic Web principles and technologies is gaining momentum in the area of science and education, where the need for efficient ways to share information and knowledge openly and globally is most obvious. New initiatives are emerging frequently, with promising prospects for new applications, as the practices of science and education are undergoing major paradigm shifts. 